\title{
Skin based Occlusion Detection and Face Recognition using Machine Learning Techniques
}

\author{
G.Suvarna Kumar \\ C.S.E Department \\ M.V.G.R.C.E \\ A.P. India
}

\author{
M.Srinadh Swamy \\ C.S.E Department \\ M.V.G.R.C.E \\ A.P. India
}

\author{
Sumit Gupta \\ C.S.E Dept. \\ M.V.G.R.C.E \\ A.P. India
}

\begin{abstract}
In this paper, a detailed experimental study of occlusion detection in the controlled environmentsbased on skin color is proposed.The image is given as an input to the face detection algorithm to detect the faces. Some faces are not detected dueto occlusion, so an occlusion detection technique is implemented to detect all the occluded faces. Those occlusions are detected using skin color of the faces. This is implemented by using circular Hough transform through plotting of circles on the faces present in the image. In order to overcome the illumination problem, extraction of local SMQT features is done. After completion of face detection, occlusions are detected based on skin color and the respective spatial locations of the image are returned.To differentiate the skin colors with other colors, SVM classifier is used. Huge datasets are collected for the purpose of training.From the image database, the occluded faces are recognized by retrieving it through spatial location. This implementation is suitable for all face detection applications in constrained environments the experiment using this technique havegiven 94\% accuracy.
\end{abstract}

\section{General Terms}

Occlusion detection, occlusion recognition, face detection, circular hough transform

\section{Keywords}

\section{SVM classifier, SMQT features}

\section{INTRODUCTION}

From the last few decades, researchers gained attention on automated face recognition system because it is one of the biggest challenging tasks to subtract the real time background from the image. If the background is same as the skin color of the humans, then the task is very complicated and it yields less accuracy. There are so many approaches to identify the faces and recognize them. The latest development in this area of work has facilitated us with high processing speed and yieldof high accuracy. There is a lot of scope in the improvement of technologies in the development of visual and computer vision system disciplines but there are some problems in improving the technologies of efficient visual feature extracting algorithms high processing power for retrieval from huge human database. Another problem is, the image database consists of different types of images like noisy images, image with background color same as skin color etc. data mining, one of the popular area of research work and also successful applications of image analysis, it will classify the different categories of images in the image database.

Over the last fifteen years, face recognition [1] is a famous research work area for computer science researchers and neuroscientists. Occlusion detection [2] and face recognition is a mandatory requirement in controlled environments like automated systems since in any automated system, it is a difficult task to detect the face recognition in a real time background world containing of a large number of datasets consisting of faces. So, we have to subtract the real time background in the image. Automated systems in the controlled environments like automated attendance system[3], where positions of students are fixed, occlusion detection and face recognition play a vital role since in case of existence of any occlusions in the images, the attendance report may contain false conclusions of declaring absentees for occluded faces even if the respective members are present. Henceforth when the occlusions existing in the image are detected and recognized, a higher level of accuracy is obtained when compared with the automated attendance system that makes uses face detection.

Face detection is a technology to determine the locations of a person in an image. It only detects the faces, rest of it is treated as background and it is subtracted from the image. In any digital images, detecting the faces as a pattern classification or in controlled environments or detection of the faces by color or detect the faces by model. By using AdaBoost and Haar cascade classifiers, we can detect the faces.

Face recognition is a technique to identify a human being from a static image or moving imageby using neural networks or by geometrical feature matching or by graph matching or by using Eigen faces, we can recognize the faces. Some other face recognition techniques are $\mathrm{PCA}[4]$ (Principal Component Analysis), LDA (Linear Discriminant Analysis), fast PCA. Occlusion detection[2] is the recovery of hidden parts of objects in a digital image by interpolation from the vicinity of occluded area. By plotting level lines, we can detect the occlusions.

\section{EXISTING SYSTEM}

One common method for detecting the faces in the image is AdaBoostclassifier[5] and Haar cascade classifier. These methods of detecting faces will give less accuracy because these methods will not work effectively if real time background is same as the skin color of the humans. For detecting occlusions, the common method used now-a-days is through plotting level lines.

To detect occlusions in an image, level lines based disocclusion involves six steps. In the first step, compute the polygonal line corresponding to the occlusion boundary, in the second step, Computation of each T-junction on the occlusion boundary. A T-junction is determined by its coordinates, its position on the boundary, the related gray levels and the average direction of the corresponding level line. In the third step, perform triangulation of the occlusion. In fourth step, Computation by dynamic programming of the optimal set of level lines pairwise connecting the $\mathrm{T}$ junctions.In the next steps, drawing of the corresponding 
geodesic paths and use of geodesic propagation to build the restored image. The complexity of level lines based occlusion detection is shown in equation 1 .

$$
\mathrm{O}\left(\mathrm{N}^{2}+\mathrm{NM}^{2}+\mathrm{M}^{3}+\mathrm{P}\right)
$$

Where $\mathrm{N}$ demotes number of vertices of the polygon line describing occlusion boundary, $\mathrm{M}$ is the number of $\mathrm{T}$ Junctions, $\mathrm{P}$ is the number of pixels in the occlusion.But, by using level lines based occlusion detection, it is not suitable for controlled environments.

To recognize the faces, now-a-days LDA is used for recognition of faces. It finds the vectors in the space that best discriminate among classes. The main aim of LDA is to maximize the scatter matrix of inter classes and minimize the intra class scatter matrix. To recognize the faces using LDA, it consists of some series of steps like calculating the average face vector and it is subtracted from the vector Y. Here vector $\mathrm{Y}$ means representing the faces in the database in terms of vector $Y$. then ,the images are categorized based on the subjects present in the image and the scatter matrix is caluculated. Using these scatter matrix, calculate the nonsingular scatter matrix. After calculating the non-singular scatter matrix, calculate its Eigen vectors and project the basis vector and weights of image. The main drawback of this technique is large amount of memory required and greater computational complexity and it yields less accuracy when this algorithm is not used along with other algorithms like PCA and fast PCA.

\section{PROPOSED SYSTEM}

The proposed method introduces a new technique to detect the occlusions in the image and recognize those faces in the controlled environments like automated attendance system. The proposed system consists of mainly three steps. First step is face detection in the given digital image, second step is to detect the occlusions in the image, and final step is to recognize those faces in the image. To implement the first step, we use the method of circular Houghtransform [6] and for implementing second step, we uses skin color based occlusion detection. For this, SVM classifier is used to classify the skin color and other colors in the image by training the datasets. To implement the final step, that is face recognition, by retrieving the image from image database through consideration of spatial locations of the occlusions. These spatial locations are the output of second step, occlusion detection. Figure 2 shows the proposed flow of implementation.

\subsection{Circular Hough Transform}

The circular Hough transform is a feature extraction technique used in computer vision, image analysis and digital image processing. The main goal of circular hough transform is to find the imperfect instances of objects within a class of shapes. This procedure is carried out in a parameter space from which object candidates are obtained as local maxima in an accumulator space that is explicitly constructed by calculating the circular hough transform.

The circular hough transform was initially concerned with the identification of lines in the image, but later the hough transform has been extended to identify the positions of arbitrary shapes, most commonly circles or ellipses.

In automated analysis of digital images, there is a problem of identifying the shapes like circles or ellipses or straight lines. In many cases, edge detector can be used as pre-processing technique to obtain image points or pixels on the desired curve in the image space. Because of the imperfections existing in the digital image, there are many missing points on the desired curves as well as spatial deviations between lines or circles or ellipses.Through the use of edge detector, noisy edge points are obtained. The main purpose of circular hough transform is to address the problem of performing groups of edge points into object candidates.

The simplest way of circular hough transform is the linear transform for detecting straight lines. In the image space, the straight line can be described as $y=m x+b$ and can be plotted graphically by using pair of image points $(\mathrm{x}, \mathrm{y})$. In the circular hough transform, the main idea is to consider characteristics of straight line, it uses slope $\mathrm{m}$ and intercept $\mathrm{b}$. Hence, the image points are represented as $(b, m)$ in the parameter space.

The circular hough transform method of plotting circles consists of segmentation process. Segmentation is the one of the challenging task, like noisy images. For this purpose, threshold or edge detection routines are used. To discard the color information, adaptive histogram equalization is used. We estimate the radii of interested range about 4 to 20 pixels by using equation 2

[accum, circen, cirrad $]=$ circularHough_Grd $([4,20])$ (2) Plot the circles on the image by using the below equation3.

Plot(circen(:,1), circen(:, 2), ' $r+$ ');

\subsection{Face detection training \&classification}

The face detector analyses image patches $32 \times 32$ pixels is applied. That patch is extracted and classified by keeping $\Delta x=1$ and $\Delta y=1$ pixels through the whole image. To find the humans in the digital image, the image is downscaled and resized with a scale factor less than 1.2. For every pixel, it contains feature vector by analyzing its vicinity. This vector is recalculated to the index, here index is the position of the feature vector. A circular mask contains $\mathrm{p}=648$ pixels applied to every patch to remove background pixels and it avoids effects of the edges from the possible filtering and ignores the undefined pixels at rotation operation. The face and non faces are trained with the parameters $\alpha=1.0051, \beta=0.9951$ and $\gamma=200.14$.

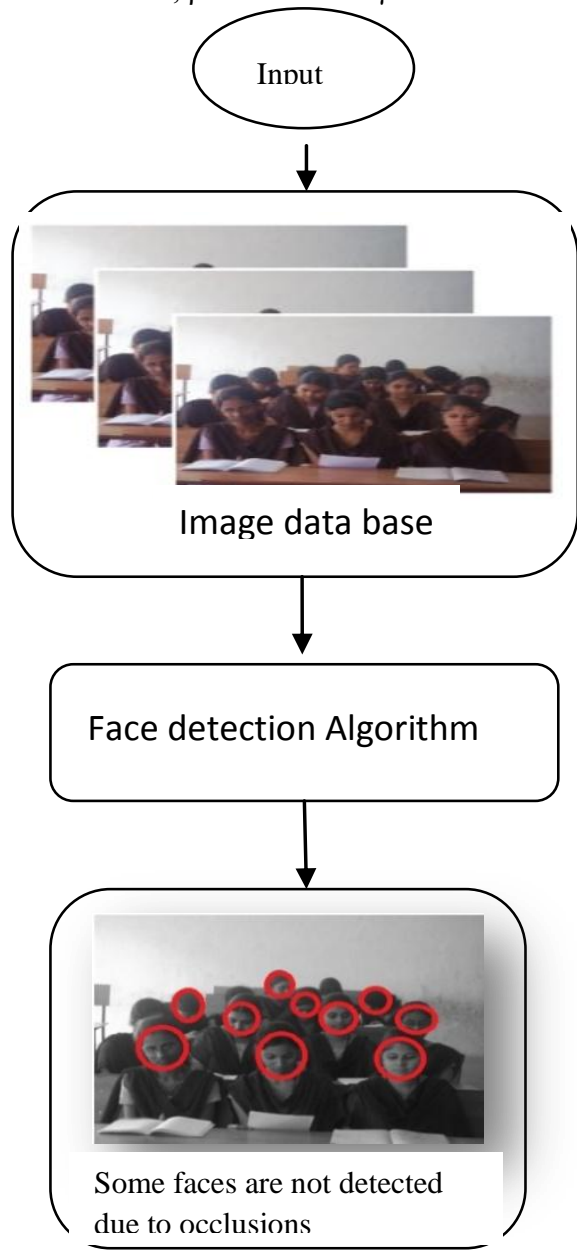




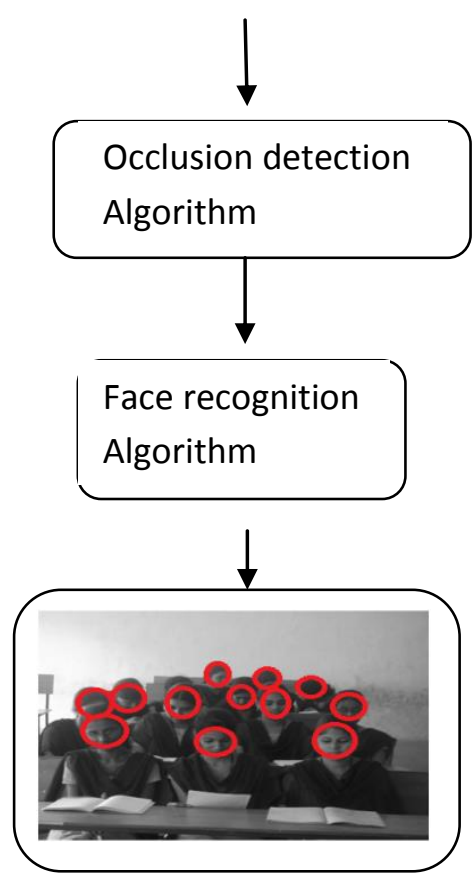

Fig2: Proposed System

\subsection{SVM Classifier}

SVM (Support Vector Machines)[7], a new method for classification for both linear and nonlinear data. In a nutshell, a support vector machines is an algorithm that works as follows.SVM criteria will minimize the overlap between classification hyper plane and sub space of solutions in class 1[8]. It uses a nonlinear mapping to transform the original training data into a higher dimension [9]. Within this new dimension, it searches for the linear optimal searching hyper plane (that is, a decision boundary separating the tuples of one class from another).

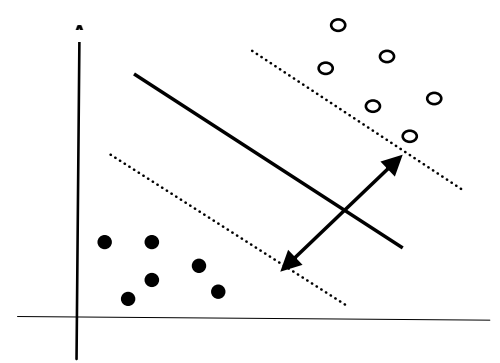

Fig 3: SVM classifier classifies two classes

In this implementation, SVM classifies the skin color and nonskin color present in the image and returns the locations where occlusions present. We can separate faces based on colors is shown in below equation 4

$$
\mathrm{w}_{0}+\mathrm{w}_{1} \mathrm{x}_{2}+\mathrm{w}_{2} \mathrm{x}_{2}=0
$$

Thus, any point that lies above the separating hyper plane satisfies

$$
\mathrm{w}_{0}+\mathrm{w}_{1} \mathrm{x}_{1}+\mathrm{w}_{2} \mathrm{x}_{2}>0
$$

Similarly, any point that lies below the separating hyper plane satisfies

$$
\mathrm{w}_{0}+\mathrm{w}_{1} \mathrm{x}_{1}+\mathrm{w}_{2} \mathrm{x}_{2}<0
$$

\subsection{Face Recognition}

Face recognition is one of the nonintrusive biometric techniques commonly used for verification and authentication. Face recognition uses the local and global features. For the global based feature extraction technique, it calculates the covariance matrix for images. For the local based feature extraction, it calculates the auto-correlation matrix. In this implementation, we recognize the faces using HSPCA [10] algorithm.

\section{RESULTS}

In this paper, experiments are run on the MATLAB to observe the effectiveness of the proposed system. We tested the proposed system with 24 images which includes college student's images and background color which is same as skin color. For each student's image, it consists of 12 students, out of 12 students, only 9 students $(78 \%)$ are detected using face detection technique. That is, in the image, the system cannot detect 3 students. By using the skin color based occlusion detection, those 3 students are detected. In the figure 3, it shows comparison between existing system and proposed system and figure 4 shows the results of face detections in different environments.

\subsection{Experiment 1}

Image consists of 12 students shown in fig 4 and that image is applied to face detection algorithm. Face detection algorithm detects 9 students out of 12 students. That is, face detection algorithm didn't detect 3 students shown in fig 5 . The output of face detection algorithm is applied to occlusion detection algorithm and it detects 2 students those are not detected by face detection algorithm. After detecting the faces, face recognition algorithm recognize the occluded faces also and it is shown in fig 6 .

\subsection{Experiment 2}

We tested the proposed system with image with background color is same as skin color. The image consists of 6 persons shown in fig 7 and image is applied to face detection algorithm, it only detects 4 persons and 2 persons are not detected by the face detection algorithm. The output of the face detection algorithm is applied to occlusion detection algorithm. After detecting the faces, face recognition algorithm recognize the faces whose skin color is same as background color and it is shown in fig8.

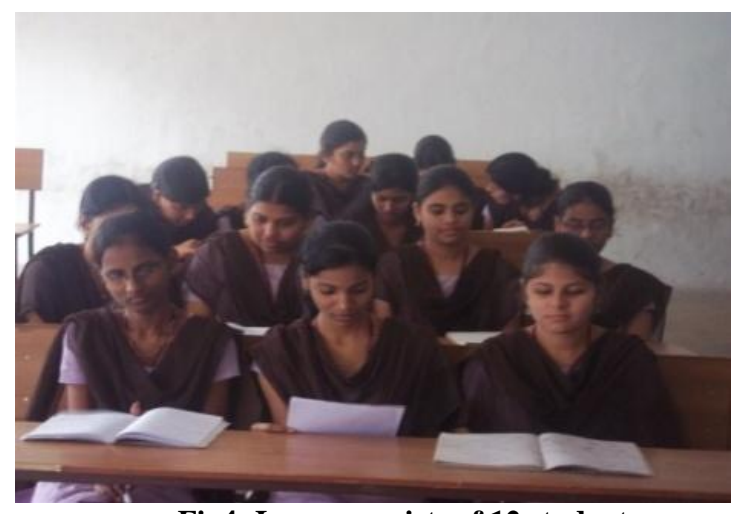

Fig4: Image consists of 12 students 


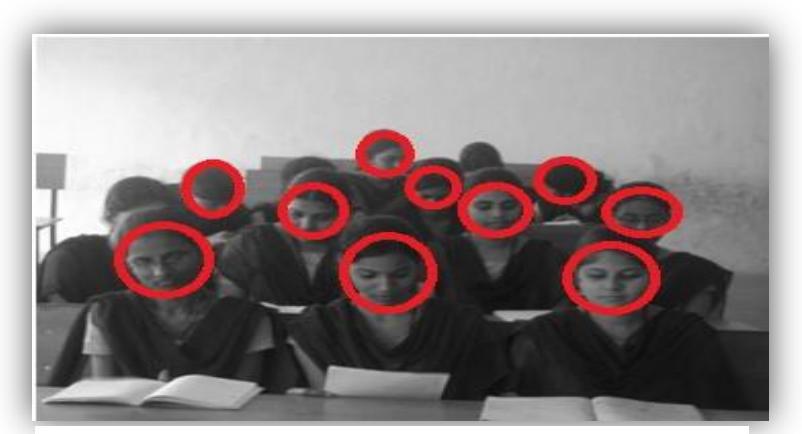

Fig 5: Output of the face detection algorithm this could not detect all faces

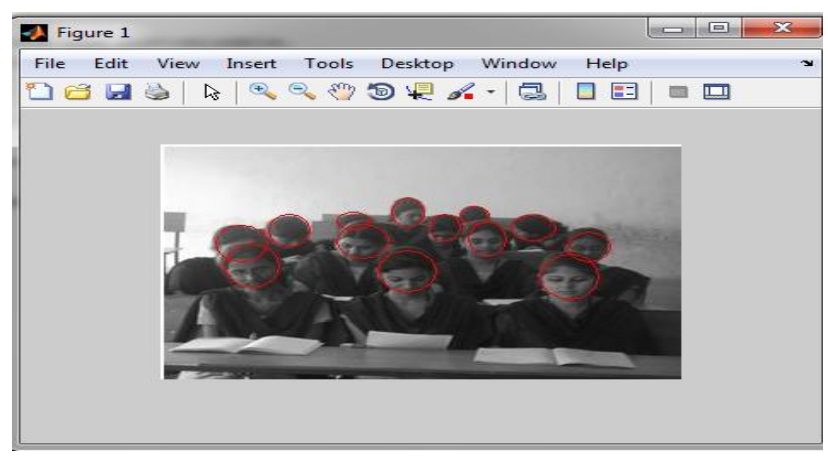

Fig6: Output of the proposed system where all the faces were detected

Table 1 shows the comparisons between face recognition algorithms in different environments and fig 9 shows the comparison between existing system and proposed system.

Table 1: Comparisonbetween face recognition algorithms in different environments

\begin{tabular}{|c|c|c|}
\hline $\begin{array}{c}\text { Images in } \\
\text { different } \\
\text { environments }\end{array}$ & $\begin{array}{c}\text { Faces detected } \\
\text { by the face } \\
\text { detection } \\
\text { algorithm }\end{array}$ & $\begin{array}{c}\text { Faces detected by } \\
\text { occlusion } \\
\text { detection } \\
\text { algorithm }\end{array}$ \\
\hline $\begin{array}{c}\text { Image of } 12 \\
\text { students }\end{array}$ & 9 & 11 \\
\hline $\begin{array}{c}\text { Image of } 6 \\
\text { persons } \\
\text { whose skin } \\
\text { color is same } \\
\text { as background } \\
\text { color }\end{array}$ & 4 & 6 \\
\hline
\end{tabular}

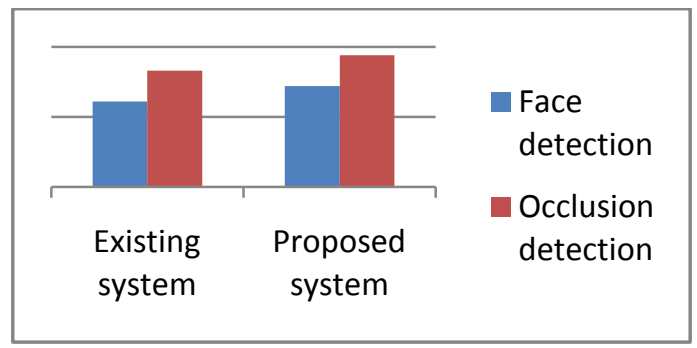

Fig 7: comparing the proposed system results with existing system results

\section{CONCLUSION}

In this paper, we implemented the occlusion detection in controlled environments and tested it with different images. Face detection in the digital image is a challenging task because it consists of real time background and sometimes background color is same as skin color. So, in this paper, detected the occlusions based on the skin color which gave more accuracy than existing systems.

\section{ACKNOWLEDGEMENTS}

Suvarna Kumar $\mathbf{G}$ is currently working as the Senior Assistant Professor in CSE Department of MVGR College of Engineering. His Research area is Speech and video processing. He published 6 international journals and attended 2 conferences in Singapore. He has 9 years teaching experience, 2 years R \&D and 1 year industrial experience. He is a member of ACM, National Science Congress, IAENG and IACSIT

Prasad Reddy P.V.G.D is currently the Rector (Pro-Vice Chancellor) of Andhra University. He completed Phd in the year 1993. He has $\mathbf{2 4}$ years of teaching \&research experience which includes more than $\mathbf{1 0}$ years of administrative experience with Andhra University at various senior capacities. He has so far successfully 20 Ph.D.'s, 18 M.Phil.'s and 259 M.Tech Projects in the field of Computer Engineering, and has to his credit, more than 104 Research papers which includes 42 publications in the referred international Journals of repute, $\mathbf{2}$ Patents filed and he has presented 19 publications in International Conferences across the world.

SrinadhSwamy M is pursuing MTech from MVGR College of engineering. His project Research Area is image processing

Sumit Gupta is currently the HOD of the CSE department in MVGR College of engineering. He Completed his PhD from NUS Singapore in Image processing and 3D Visualization research area. He has 8 years teaching experience, 11 years $\mathrm{R}$ \& D and 3 years industry experience. He has 39 publications which include 8 national journals, 11 international journals, 11 national conferences and 9 international conferences. He was a member of professional bodies like CSI and ACM.

\section{REFERENCES}

[1] G.Suvarna Kumar, P.V.G.D Prasad Reddy, R.Anil Kumar, Sumit Gupta, "Position Detection with Face Recognition using Image Processing and Machine Learning Techniques," in IJCA special issue on Novel aspects of Digital Image Applications, DIA 2011

[2] C. Lawrence Zitnick, Takeo Kanade, "A Cooperative Algorithm for Stereo Matching and Occlusion Detection" CMU-RI-TR-99-35.

[3] Yohei Kawaguchi, Tetsuo Shoji, Weijane Lin, KohKakusho,Michihiko Minoh, "Face Recognition based Attendance System" http://www.mm.media.kyotou.ac.jp .

[5] P. Viola and M. Jones, "Rapid object detection using a boosted cascade of simple features," in In Proceedings of the 2001IEEE Computer Society Conference on Computer Vision andPattern Recognition (CVPR), 2001, vol. 1, pp. 511-518. 
[6] Mohamed Rizon Haniza, Yazid Puteh, Saad Ali Yeon, Md Shakaff," Object detection using circular hough transforms"

[7] E. Osuna, R. Freund, and F. Girosi, "Training support vector machines:an application to face detection," in Proceedings of the IEEE Conference on Computer Vision and Pattern Recognition (CVPR '97), 1997, pp. 193-199.
[8] Hongjun Jia and Aleix M. Martinez, "Support Vector Machines in Face Recognition with Occlusions", Computer Vision and Pattern Recognition - CVPR , pp. 136-141, 2009

[9] Jiawei Han,MichelineKamber, Jian pei "Data mining : concepts and techniques" third edition

[10] Niranjan, M. "Support vector machines: a tutorial overview and critical appraisal" in Applied Statistical Pattern Recognition (Ref. No. 1999/063), IEE Colloquium on, 20 Apr 1999, ref number 1999/063. 